\title{
The developmental course of inattention symptoms predicts academic achievement due to shared genetic aetiology: A longitudinal twin study
}

Liu, Chao-Yu, M.D., M.S. ${ }^{1,2}$, Li, Yan, M.S. ${ }^{3}$, Viding, Essi, Ph.D. ${ }^{1}$, Asherson, Philip, MRCPsych, Ph.D. ${ }^{4}$; Pingault, Jean-Baptiste, Ph.D. ${ }^{1,4}$

${ }^{1}$ Department of Clinical, Educational and Health Psychology, University College London, London, United Kingdom.

${ }^{2}$ Department of Psychiatry, National Taiwan University Hospital and College of Medicine, Taipei, Taiwan.

${ }^{3}$ Faculty of Psychology, Beijing Normal University, Beijing, China.

${ }^{4}$ Social, Genetic \& Developmental Psychiatry Centre, King's College London, London, United Kingdom.

**Address for correspondence:

Division of Psychology and Language Sciences, University College London, 26 Bedford Way, London, WC1H 0AP, UK. j.pingault@ucl.ac.uk, +44(0)2076792000

Key words: ADHD; Inattention symptoms; Academic achievement; Twins; Genetic and environmental aetiology

Word count: 3393

\section{Acknowledgements}

We gratefully acknowledge the ongoing contribution of the participants in the Twins Early Development Study and their families. The Twins Early Development Study is supported by grant G0901245 (and previously G0500079) from the UK Medical Research Council. E.V. is supported by a Royal Society Wolfson Research Merit Award from the Royal Society and the Wolfson Foundation. JB.P. is a fellow of MQ Transforming Mental Health (MQ16IP16). CY.L. is supported by Overseas Research Scholarship from University College London. 


\section{Abstract}

\section{Background}

Symptoms of Attention-Deficit Hyperactivity Disorder, in particular inattention symptoms, are associated with academic achievement. However, whether and why the developmental course of inattention symptoms (i.e. systematic decreases or increases of symptoms with age) predicts academic achievement remains unclear.

\section{Method}

A total of 5634 twin pairs born in the UK were included in the current study. We used Latent growth curve modelling to: estimate the baseline level and the developmental course of inattention symptoms (assessed at ages 8, 11, 14 and 16 years) and test whether they predicted the General Certificate of Secondary Education scores (GCSE, at age 16 years). We then implemented Multivariate twin modelling to determine the role of genetic and environmental factors in explaining the relationship between inattention symptoms and GCSE scores.

Results Increasing inattention symptoms across childhood and adolescence predicted poorer GCSE scores independently of the baseline level of inattention. Genetic factors explained most of this relationship, i.e. genetic factors contributing to individual differences in the developmental course of inattention also influenced GCSE scores.

\section{Conclusion}

Genetic factors underlying the developmental course of inattention symptoms across childhood and adolescence also influence academic achievement. This may result from indirect mechanisms whereby genetic factors explain systematic changes in inattention levels 
with age, which in turn impact academic achievement. The shared genetic aetiology may also suggest common neurobiological processes underlying both the developmental course of inattention symptoms and academic achievement. 


\section{Introduction}

Attention-deficit hyperactivity disorder (ADHD) is a highly heritable neurodevelopmental disorder characterised by two distinct symptom dimensions: inattention and hyperactivity/impulsivity [1]. The heritability of ADHD symptoms is estimated to be around $80 \%[2,3]$. Genetic factors also independently contribute to the developmental course of ADHD symptoms across the lifespan, i.e. systematic increase, decrease or persistence of symptoms with age $[4,5]$. Although twin studies report a high genetic correlation $(0.55-0.62)$ between inattention and hyperactivity/impulsivity, suggesting overlapping genetic influences underlying the two symptom dimensions, they also identify genetic effects specific to each symptom dimension. The findings support partially distinct aetiology underlying the two ADHD symptom dimensions and encourage researchers to investigate the two dimensions separately in quantitative genetic analyses [6-8].

ADHD is a major source of functional impairment $[9,10]$. Among these, academic underachievement is a common difficulty experienced by children with ADHD [11, 12]. Notably, the two ADHD symptom dimensions contribute differentially to academic outcomes [13]. Inattention directly predicts academic achievement as it specifically predicts learning difficulties such as mathematics, reading and spelling ability [14, 15]. Conversely, hyperactivity/impulsivity is less strongly associated with learning ability but is associated with externalising behaviours, classroom disruption and peer relational problems [16]. The unique influence of inattention symptoms on academic achievement across developmental stages was confirmed in systematic reviews and meta-analyses [17-19]. The significant phenotypic relationship between inattention symptoms and academic achievement could be partly attributable to a shared genetic aetiology. For example, a genetic correlation of -0.31 was found between inattention and reading difficulty and -0.41 between inattention and 
mathematics ability. Conversely, the genetic correlation between hyperactivity/impulsivity and learning ability was significantly lower at -0.12 with reading ability and -0.22 with mathematics ability respectively [20]. The developmental course of the two ADHD symptom dimensions also predicts academic achievement differently. Results from two population samples in the United States and Canada showed that increasing inattention levels from childhood to adolescence significantly predicted high school graduation failure and poorer mathematics and reading abilities [21, 22]. Furthermore, the developmental course of inattention symptoms independently predicted academic outcomes regardless of average symptom severity [22]. On the contrary, trajectories reflecting the developmental course of hyperactivity/impulsivity symptoms did not predict academic achievement when inattention symptoms were controlled for [23]. Taken together, the above findings support the unique influence of both baseline level and the developmental course of inattention symptoms on academic achievement. However, replication of these findings in different population samples is needed. In addition, given the high heritability of academic achievement [24], whether the unique influence of the developmental course of inattention on academic achievement is attributable to a common genetic and environmental aetiology needs further exploration.

To further examine the relationship between inattention symptoms and academic achievement, we applied twin modelling to a population-based cohort comprising 4 waves of assessment of inattention symptoms at ages 8,11, 14 and 16 years. Academic achievement was indexed, at age 16 years, by scores on the General Certificate of Secondary Education (GCSE), a nationwide examination at the end of compulsory schooling in the United Kingdom. We hypothesised that: 1) the developmental course of inattention symptoms would influence GCSE scores at age 16 years independently of the baseline level of symptoms; 2) genetic factors influencing the baseline level and the developmental course of inattention 
symptoms would also partly explain their contribution to GCSE scores.

\section{Methods}

\section{Participants}

Participants were drawn from the Twins Early Development Study (TEDS), a longitudinal study of twins recruited from population birth records in England and Wales between January 1994 and December 1996 [25, 26]. The current study sample included twin pairs for whom both twins had GCSE scores at the end of compulsory schooling and at least one ADHD symptom assessment between ages 8 and 16 years. Missing GCSE scores can be due to study attrition, family who chose not to report GCSE results, twins with special educational arrangements and school drop-outs. The mean level of inattention ratings was higher in twin pairs without GCSE scores, with Cohen d ranging from 0.24 to 0.38 (Supplementary Table 1). Twins with pre- or perinatal complications, severe congenital anomalies, autistic disorder, chromosomal disorders, and those who failed to provide zygosity information were excluded. A total of 5634 twin pairs were included in the current analysis, of which $53.3 \%$ were female. The study sample was adequately representative of the UK population as compared with the UK census data from the general household survey (Supplementary Table 2). Approval was obtained from the Institute of Psychiatry, Psychology and Neurosciences Ethics Committee at King's College London Psychology and Neuroscience Department. Written informed consent was acquired from parents prior to data collection. All human and animal studies have been approved by the appropriate ethics committee and have therefore been performed in accordance with the ethical standards laid down in the 1964 Declaration of Helsinki and its later amendments. 


\section{Measures}

Inattention symptoms were assessed with the Conners' Parent Rating Scales-Revised (CPRSR) at age 7.9, 11.3, 14.2 and 16.3 years. The CPRS-R consists of two subscales: inattention and hyperactivity/impulsivity [27]. Each subscale comprises nine statements that describe ADHD symptoms based on the DSM-IV criteria, evaluated on a Likert rating scale with four levels from "not true at all" (0) to "very much true" (3). Higher scores indicate greater severity. From this dataset, standardised Cronbach alphas across the four ages were found to range between 0.87 and 0.90 for inattention and 0.77 and 0.83 for hyperactivity/impulsivity Academic achievement was assessed using scores from the General Certificate of Secondary Education (GCSE) at age 16 years. Data were collected by telephone interviews and mail questionnaires to the twins and their parents. The reliability of self-reported GCSE results was confirmed by comparing them with data from the National Pupil database (correlation of 0.98 for English, 0.99 for mathematics, and 0.96 for all sciences) [28]. The overall GCSE mean score was the average of the three core subjects: mathematics, English and science.

\section{Statistical Analysis}

The literature reviewed in the introduction shows that inattention symptoms are more important than hyperactivity/impulsivity in predicting academic achievement, hence, we focused on analysing the nature of the contribution of inattention symptoms to academic achievement. Findings for hyperactivity/impulsivity are presented in the supplementary material for interested readers. All scores were regressed on age and gender prior to analyses. 
First, a latent growth curve model was built to capture the baseline level (intercept) and the developmental course (slope, systematic decreases or increases in symptoms from age 8 to 16 years) of inattention symptoms from childhood to adolescence. This model was then expanded to include regression parameters to investigate the effects of the intercept and the slope of inattention symptoms on GCSE scores at age 16, setting GCSE scores as the dependent (outcome) variable. Relevant parameter constraints were imposed in the latent growth model to account for non-independence between twins following the procedure described in Olsen and Kenny for exchangeable dyads [29].

Second, the latent growth models without and with GCSE estimated in the first step were fully developed into multivariate genetic models in order to: (i) examine genetic and environmental influences on the baseline level and the developmental course of inattention, and (ii) determine how much of the variance in GCSE scores was attributable to genetic and environmental components underlying the baseline and the developmental course of inattention symptoms. We used the standard Cholesky decomposition [30] to estimate the relative contributions of additive genetic (A), dominant genetic (D), shared environment (C) and non-shared environmental (E) factors to the baseline level and developmental course of inattention symptoms. The $\mathrm{C}$ component encompasses the environmental factors that make the twins within a pair similar. The E component captures any environmental factors that make the twins different, and also includes measurement error. Whether dominant genetic effects (D) are estimated depends on the monozygotic (MZ) to dizygotic (DZ) within-pair correlations. If the within-pair correlation in MZ twins is more than twice higher than the within-pair correlation in DZ twins, then dominant genetic effects can be present and estimated. In a previous study using the same sample, the best-fitted genetic model for inattention symptoms was an ADE model [5], where dominant genetic effects (D) explained 
$55 \%$ and $35 \%$ of the variance in the intercept and slope of inattention symptoms. ACE model fitted best for GCSE scores and shared-environmental factors (C) explained $26 \%$ of the variance in GCSE [24]. Given the discrepancy in best-fitting models for inattention symptoms and GCSE, and the need to model them simultaneously to answer our research questions, we estimated an AE model for inattention symptoms, where the A component reflected broad sense heritability (both additive genetic effects and non-additive genetic effects). An ACE model was used for GCSE.

The goodness-of-fit of the latent growth curve models were assessed using the comparative fit index (CFI), root mean square error of approximation (RMSEA) and the standardized root mean square residual (SRMR). A model is considered as an adequate fit when CFI $\geq 0.90$, RMSEA $\leq 0.06$ and SRMR $\leq 0.08$ [31-33]. All analyses were performed using R software version 3.2.3 [34] using a built-in structural equation modelling package lavaan version 0.520 [35]. A maximum likelihood estimator was used to deal with missing data, while $95 \%$ confidence intervals were obtained by bootstrapping (number of bootstrap replicates $=5000$ ).

\section{Results}

Descriptive statistics for inattention symptoms, GCSE scores and their age-to-age withintwin/cross-twin phenotypic correlations are presented in Table 1 for MZ twins and Table 2 for DZ twins. As shown in the table, the mean level of inattention decreased slightly from age 8 to age 16 years and inattention scores were negatively correlated with GCSE scores. Significant zygosity differences were found in the cross-twin cross-trait phenotypic correlations between inattention and GCSE scores, suggesting that genetic factors contribute to the relationship between inattention symptoms and GCSE scores. Findings for hyperactivity/impulsivity symptoms are displayed in Supplementary Table 3. 


\section{Modelling the developmental course of inattention}

The estimated intercept of inattention symptoms was 5.26 (95\% CI 5.14, 5.38), representing the baseline scores on the CPRS-R inattention scale at age 8 years. The estimated slope, reflecting systematic change in inattention symptom scores across follow-up (i.e. the developmental course), was estimated to -1.12 (95\% CI -1.28, -0.95). This negative slope corresponded to a systematic decrease of 1.12 point in the CPRS-R inattention scale per decade, leading to a predicted score of 4.13 at the end of the follow-up. The contributions of both the baseline level $(\mathrm{B}=-0.13,95 \% \mathrm{CI}(-0.14,-0.12)$ and the developmental course $(\mathrm{B}=-0.08,95 \% \mathrm{CI}(-0.09,-0.07)$ of inattention to GCSE scores were significant. The findings suggest that a 1-point higher initial level and 1-point higher slope of inattention symptoms were associated with, respectively, 0.13 - and 0.08 -point decrease in GCSE scores.

\section{Multivariate genetic model}

\section{AE model for inattention}

Table 3 presents the standardised genetic and environmental influences on inattention symptoms in the AE Cholesky model. The broad heritability of inattention was high across ages with $75 \%$ to $80 \%$ of the total variance explained by genetic factors. Genetic innovation (i.e. genetic factors emerging with age) explained $32 \%$ to $36 \%$ of the variance of inattention symptoms at each age. The non-shared environment (total $\mathrm{e}^{2}$ ) accounted for $20 \%$ to $25 \%$ of the total variance of inattention symptoms (Table 3). Continuity of the non-shared environmental influences from early environmental factors were small, ranging from only $1 \%$ to $5 \%$. Findings for hyperactivity/impulsivity symptoms are displayed in Supplementary 
Table 4.

\section{Shared aetiology between inattention and GCSE scores}

Figure 1 presents the model examining to what extent the genetic and the environmental factors underlying inattention symptoms contributed to GCSE scores. The fit indexes for the inattention model was good: $\mathrm{CFI}=0.97, \mathrm{RMSEA}=0.038, \mathrm{SRMR}=0.077$.

As shown in Figure 1, the initial genetic factors (A1) explained 82\% (95\%CI: 78\%-85\%) of the variance in the baseline level and $10 \%$ (95\%CI 6\%-15\%) of the variance in the developmental course of inattention symptoms. The newly identified genetic factor A2 explained 54\% of the variance in the developmental course of inattention independently of A1. A1 and A2 respectively explained 10\% (95\%CI 8\%-12\%) and 5\% (95\%CI 3\%-7\%) of the variance in GCSE scores. In contrast to the findings for genetic factors, the initial environmental factor (E1) and the newly identified environmental factor (E2) only accounted for a total of $2 \%$ variance in GCSE scores. Overall, the genetic factors underlying the intercept and the slope of inattention symptoms explained $14.8 \%$ of the phenotypic variance of GCSE and $25.7 \%$ of the heritability of GCSE (see caption of Figure 1). Findings expressed in terms of correlations were as follow: the phenotypic correlation between the intercept and GCSE was -0.32 and could be decomposed into a genetic component $(-0.28 ; 88 \%$ of the phenotypic correlation) and a non-shared environmental component $(-0.04 ; 12 \%$ of the phenotypic correlation). Similarly, the phenotypic correlation between the slope and GCSE was -0.24 and could be decomposed into a genetic component $(-0.18 ; 74 \%$ of the phenotypic correlation) and a non-shared environmental component $(-0.06 ; 26 \%$ of the phenotypic correlation). The Cholesky decomposition of GCSE is also presented in the caption of Figure 1. 
As expected, the baseline level and the developmental course of hyperactivity/impulsivity were less predictive of GCSE scores (Supplementary Figure 1). 


\section{Discussion}

The current study examined to what extent the baseline level and the developmental course of inattention symptoms predicted academic achievement, indexed as GCSE scores at age 16 years, in a large population-based twin cohort. Results showed that the baseline level and the developmental course of inattention symptoms from childhood to adolescence significantly and independently predicted GCSE scores. The predictive effects mainly resulted from underlying genetic influences: genetic factors contributing to the baseline level and the developmental course of inattention symptoms collectively explained $15 \%$ of the individual differences in GCSE scores.

\section{Inattention symptoms and academic achievement}

Our finding that higher baseline inattention symptoms predicted lower long-term academic achievement was consistent with results from multiple previous studies $[13,15,36]$. Importantly, we also replicated the findings from two previous longitudinal studies highlighting the independent role of the developmental course of inattention symptoms in long-term academic achievement $[21,22]$. In the current study, we added to the growing body of evidence that systematic change in inattention symptoms across childhood and adolescence contributes to long-term functional outcomes.

\section{Genetic and environmental underlying factors}

This study is the first to examine the genetic and environmental aetiology underlying the contribution of both the baseline level and the developmental course of inattention symptoms to academic achievement. We found that genetic factors contributing to the baseline level and the developmental course of inattention symptoms accounted for $15 \%$ of the phenotypic variance of GCSE and explained a quarter of the heritability of GCSE. These findings 
suggest that developmental genetic effects underlying systematic changes in inattention symptoms across childhood and adolescence also contribute to academic achievement.

This genetic overlap between the developmental course of inattention and academic achievement can be explained in two (non-exclusive) ways: a causal effect of inattention on academic achievement and a shared aetiology. First, the genetically driven phenotypic change in inattention symptoms can contribute to individual differences in academic achievement. In other words, genetic factors contribute to increasing inattention symptoms across childhood and adolescence, which then compromise learning abilities and lead to lower academic achievement. Importantly, this putative causal relationship between inattention symptoms and academic achievement still awaits to be clearly established. As shown in the study by de Zeeuw and colleagues [37], although a causal effect of inattention symptoms on academic achievement after accounting for confounding genetic and environmental influences was identified, the possibility of reverse causation, whereby poor academic performance exacerbated attentional problems could not be ruled out. Moreover, research investigating causal relationships between the developmental course of inattention and academic achievement is still lacking. Research with designs such as Mendelian randomisation [38, 39] may help to estimate putative bidirectional effects between inattention and academic achievement. Longitudinal fixed-effect models could test whether within-individual changes in inattention symptoms affect changes in academic achievement [40].

Second, common genetic factors that influence neurobiological structure and functioning may underlie both the developmental course of inattention symptoms and academic achievement (genetic pleiotropy). As a plausible candidate for such shared neurobiological processes, studies found that individuals with persistent ADHD symptoms exhibited more rapid thinning in frontal brain area supporting attention and cognitive control from childhood to adulthood 
[41][Shaw, $2013 \# 235]$. The same brain regions are also involved in the development of cognitive ability during childhood and adolescence [42].

Noticeably, the genetic influence underlying inattention symptoms only accounted for $15 \%$ the total variance of GCSE scores, leaving a majority of the variance in GCSE scores accounted by other factors. This may explain findings that a treatment strategy solely targeting ADHD core symptoms only partially improve learning ability or ultimate academic attainment $[43,44]$. Therefore, multimodal interventions, consisting of ADHD symptom reduction, parenting skill training, child social skill training and behavioural management training at school could prove valuable to improve educational outcomes [45].

Non-shared environmental factors played a negligible role in explaining the relationship between inattention symptoms and GCSE scores, despite significant non-shared environmental influences on the developmental course of inattention symptoms. These findings are consistent with: (i) ample evidence of innovative non-shared environmental influences, emerging at different developmental stages, for both inattention and cognitive ability [46, 47]; (ii) evidence that the early non-shared environmental influences on inattention barely influence inattention itself at later ages [5, 48]; and (iii) previous evidence of a small overlap between non-shared environmental components of ADHD symptoms and educational outcomes [49]. Consequently, our findings that the non-shared environmental component of the baseline levels and the slope of inattention symptoms play a minor role in explaining later GCSE results appear unsurprising. Such findings may be expected considering that children are constantly under transition from different schools, teachers, peers, curricula and so forth. Hence, such environmental factors may be more time-specific by nature [50]. Nevertheless, one meta-analysis [51] found that the contribution of the non- 
shared environment to specific cognitive ability progressively increased with age and reached its maximum in late adulthood, accompanied by a gradual decrease in shared-environmental influences; these findings suggest that such time-specific factors may still play an important role in the aetiology of cognitive ability. Importantly, estimates of non-shared environmental influences should be interpreted with caution for two reasons: they include measurement error and can vary substantially by informants and assessment measures [52] [46]. Therefore, future research with a longitudinal design from childhood to adulthood which incorporates reports from multiple informants may enable a more accurate estimation of the role of the non-shared environment in the relationship between inattention symptoms and academic achievement.

\section{Limitations}

First, twin pairs with incomplete GCSE score records were excluded to estimate the relationship between inattention symptom development and academic achievement. Since inattention ratings were higher in individuals without GCSE scores, this could possibly underestimate the contribution of inattention symptoms to academic achievement.

Second, inattention symptoms were measured through parent reports. The use of parental report ensured consistent assessment throughout development, which allowed us to estimate systematic change from childhood to adolescence. However, heritability estimates in quantitative genetic studies are often subject to informant-related issues [2]. In particular the heritability of ADHD as derived from parent reports tends to be higher than that from selfreports [53]. Therefore, replication of the current findings using multiple informants is warranted. 
Third, the study used questionnaire-based rating scales to assess the severity of inattention symptoms in a population sample. Such a dimensional approach is not equivalent to the categorical clinical diagnosis and the results may not directly apply to clinical populations. However, converging evidence shows that the genetic overlap between questionnaire-rating and DSM diagnosis of ADHD is high [54, 55]. Shared genetic risk between individuals with ADHD diagnosis and their siblings was dimensionally distributed and proportional to the reported ADHD trait scores [56]. Similarly, polygenic risk scores for ADHD diagnosis could predict ADHD traits in the general population [57], suggesting the presence of a common genetic liability.

Finally, this study suffers from the common assumptions and limitations of the classical twin method, such as equal environment assumption and generalisability to the rest of the population. Although twin methods have been used extensively to study the aetiology of ADHD and other phenotypes, our results still should be interpreted with caution.

In conclusion, the genetic factors contributing to the developmental course of inattention symptoms across childhood and adolescence also influence long-term academic achievement. Our results highlight the importance of early detection and management for young children with inattention symptoms, as well as the need to monitor the change of symptom severity across development. To curb the risk of poor academic achievement, development-sensitive multimodal interventions targeting inattention symptoms and co-developing cognitive and behavioural deficits may benefit individuals in need. 


\section{Declaration of interest}

King's College London received payments for consultancy, speaker fees, or educational and research awards from Shire, Flynn, Eli-Lilly, Janssen and Novartis for work conducted by P.A.. P.A. receives educational/research awards from Shire, Lilly, QbTech and is speaker at sponsored events for Shire, Lilly and Novartis. 


\section{Reference}

1. American Psychiatric Association and American Psychiatric Association. DSM-5 Task Force, Diagnostic and statistical manual of mental disorders: DSM-5. 2013: Washington, D.C: American Psychiatric Association.

2. Nikolas, M.A. and S.A. Burt, Genetic and environmental influences on ADHD symptom dimensions of inattention and hyperactivity: a meta-analysis. $\mathrm{J}$ Abnorm Psychol, 2010. 119(1): p. 1-17.

3. Larsson, H., et al., The heritability of clinically diagnosed attention deficit hyperactivity disorder across the lifespan. Psychol Med., 2014. 44(10): p. 2223-9. doi: 10.1017/S0033291713002493. Epub 2013 Oct 10.

4. Larsson, J.O., H. Larsson, and P. Lichtenstein, Genetic and environmental contributions to stability and change of ADHD symptoms between 8 and 13 years of age: a longitudinal twin study. J Am Acad Child Adolesc Psychiatry, 2004. 43(10): p. $1267-75$.

5. Pingault, J.B., et al., Genetic and Environmental Influences on the Developmental Course of Attention-Deficit/Hyperactivity Disorder Symptoms From Childhood to Adolescence. JAMA Psychiatry, 2015. 72(7): p. 651-8.

6. McLoughlin, G., et al., Genetic support for the dual nature of attention deficit hyperactivity disorder: substantial genetic overlap between the inattentive and hyperactive-impulsive components. J Abnorm Child Psychol, 2007. 35(6): p. 9991008.

7. Greven, C.U., F.V. Rijsdijk, and R. Plomin, A twin study of ADHD symptoms in early adolescence: hyperactivity-impulsivity and inattentiveness show substantial genetic overlap but also genetic specificity. J Abnorm Child Psychol, 2011. 39(2): p. 265-75. 
8. Kuntsi, J., et al., The separation of ADHD inattention and hyperactivity-impulsivity symptoms: pathways from genetic effects to cognitive impairments and symptoms. $\mathrm{J}$ Abnorm Child Psychol, 2014. 42(1): p. 127-36.

9. Faraone, S.V., et al., The worldwide prevalence of ADHD: is it an American condition? World Psychiatry., 2003. 2(2): p. 104-13.

10. Polanczyk, G., et al., The worldwide prevalence of ADHD: a systematic review and metaregression analysis. Am J Psychiatry, 2007. 164(6): p. 942-8.

11. Biederman, J., et al., A prospective 4-year follow-up study of attention-deficit hyperactivity and related disorders. Arch Gen Psychiatry., 1996. 53(5): p. 437-46.

12. Loe, I.M. and H.M. Feldman, Academic and educational outcomes of children with ADHD. J Pediatr Psychol., 2007. 32(6): p. 643-54. Epub 2007 Jun 14.

13. Garner, A.A., et al., The relationship between ADHD symptom dimensions, clinical correlates, and functional impairments. J Dev Behav Pediatr., 2013. 34(7): p. 469-77. doi: 10.1097/DBP.0b013e3182a39890.

14. Marshall, R.M., et al., Academic underachievement in ADHD subtypes. J Learn Disabil., 1997. 30(6): p. 635-42.

15. Massetti, G.M., et al., Academic achievement over 8 years among children who met modified criteria for attention-deficit/hyperactivity disorder at 4-6 years of age. $\mathrm{J}$ Abnorm Child Psychol., 2008. 36(3): p. 399-410. Epub 2007 Oct 17.

16. Barkley, R.A., et al., Young adult outcome of hyperactive children: adaptive functioning in major life activities. J Am Acad Child Adolesc Psychiatry., 2006. 45(2): p. 192-202.

17. Frazier, T.W., et al., ADHD and achievement: Meta-analysis of the child, adolescent, and adult literatures and a concomitant study with college students. Journal of learning disabilities, 2007. 40(1): p. 49-65. 
18. Polderman, T.J., et al., A systematic review of prospective studies on attention problems and academic achievement. Acta Psychiatrica Scandinavica, 2010. 122(4): p. $271-284$.

19. Daley, D. and J. Birchwood, ADHD and academic performance: why does ADHD impact on academic performance and what can be done to support ADHD children in the classroom? Child Care Health Dev, 2010. 36(4): p. 455-64.

20. Greven, C.U., et al., Evidence for shared genetic risk between ADHD symptoms and reduced mathematics ability: a twin study. J Child Psychol Psychiatry, 2014. 55(1): p. $39-48$.

21. Breslau, N., et al., Change in teachers' ratings of attention problems and subsequent change in academic achievement: a prospective analysis. Psychol Med, 2010. 40(1): p. $159-66$.

22. Pingault, J.B., et al., The developmental course of childhood inattention symptoms uniquely predicts educational attainment: a 16-year longitudinal study. Psychiatry Res, 2014. 219(3): p. 707-9.

23. Pingault, J.B., et al., Childhood trajectories of inattention and hyperactivity and prediction of educational attainment in early adulthood: a 16-year longitudinal population-based study. Am J Psychiatry, 2011. 168(11): p. 1164-70.

24. Krapohl, E., et al., The high heritability of educational achievement reflects many genetically influenced traits, not just intelligence. Proc Natl Acad Sci U S A, 2014. 111(42): p. 15273-8.

25. Oliver, B.R. and R. Plomin, Twins' Early Development Study (TEDS): a multivariate, longitudinal genetic investigation of language, cognition and behavior problems from childhood through adolescence. Twin Res Hum Genet, 2007. 10(1): p. 96-105. 
26. Haworth, C.M., O.S. Davis, and R. Plomin, Twins Early Development Study (TEDS): a genetically sensitive investigation of cognitive and behavioral development from childhood to young adulthood. Twin Res Hum Genet, 2013. 16(1): p. 117-25.

27. Conners, C.K., et al., The revised Conners' Parent Rating Scale (CPRS-R): factor structure, reliability, and criterion validity. J Abnorm Child Psychol, 1998. 26(4): p. 257-68.

28. Rimfeld, K., et al., Pleiotropy across academic subjects at the end of compulsory education. Sci Rep, 2015. 5: p. 11713.

29. Olsen, J.A. and D.A. Kenny, Structural equation modeling with interchangeable dyads. Psychol Methods, 2006. 11(2): p. 127-41.

30. Neale, M.C. and L.R. Cardon, Data Summary, in Methodology for Genetic Studies of Twins and Families. 1992, Springer Netherlands: Dordrecht. p. 35-53.

31. Bentler, P.M., Comparative fit indexes in structural models. Psychol Bull, 1990. 107(2): p. 238-46.

32. Hu, L.-t. and P.M. Bentler, Fit indices in covariance structure modeling: Sensitivity to underparameterized model misspecification. Psychological methods, 1998. 3(4): p. 424.

33. Jöreskog, K.G., et al., Analysis of Covariance Structures [with Discussion and Reply]. Scandinavian Journal of Statistics, 1981. 8(2): p. 65-92.

34. R Core Team, $R$ : A language and environment for statistical computing. 2014, R Foundation for Statistical Computing.

35. Rosseel, Y., lavaan: An R Package for Structural Equation Modeling. Journal of Statistical Software, 2012. 48(2): p. 1-36. 
36. Pastura, G.M., P. Mattos, and A.P. Araujo, Academic performance in ADHD when controlled for comorbid learning disorders, family income, and parental education in Brazil. J Atten Disord, 2009. 12(5): p. 469-73.

37. de Zeeuw, E.L., et al., Attention deficit hyperactivity disorder symptoms and low educational achievement: Evidence supporting a causal hypothesis. Behavior genetics, 2017. 47(3): p. 278-289.

38. Davey Smith, G. and G. Hemani, Mendelian randomization: genetic anchors for causal inference in epidemiological studies. Hum Mol Genet, 2014. 23(R1): p. R8998.

39. Pingault, J.-B., et al., Using genetic data to strengthen causal inference in observational research. Nature Reviews Genetics, 2018.

40. Gunasekara, F.I., et al., Fixed effects analysis of repeated measures data. Int J Epidemiol, 2014. 43(1): p. 264-9.

41. Shaw, P., et al., Development of cortical asymmetry in typically developing children and its disruption in attention-deficit/hyperactivity disorder. Arch Gen Psychiatry, 2009. 66(8): p. 888-96.

42. Darki, F. and T. Klingberg, The role of fronto-parietal and fronto-striatal networks in the development of working memory: a longitudinal study. Cereb Cortex, 2015. 25(6): p. $1587-95$.

43. Hechtman, L., G. Weiss, and T. Perlman, Young adult outcome of hyperactive children who received long-term stimulant treatment. J Am Acad Child Psychiatry, 1984. 23(3): p. 261-9.

44. Powers, R.L., et al., Stimulant treatment in children with attentiondeficit/hyperactivity disorder moderates adolescent academic outcome. J Child Adolesc Psychopharmacol, 2008. 18(5): p. 449-59. 
45. Enns, J.E., et al., A Multimodal Intervention for Children with ADHD Reduces Inequity in Health and Education Outcomes. Can J Psychiatry, 2017. 62(6): p. 403412.

46. Kan, K.J., et al., Genetic and environmental stability in attention problems across the lifespan: evidence from the Netherlands twin register. J Am Acad Child Adolesc Psychiatry, 2013. 52(1): p. 12-25.

47. Hoekstra, R.A., M. Bartels, and D.I. Boomsma, Longitudinal genetic study of verbal and nonverbal IQ from early childhood to young adulthood. Learning and Individual Differences, 2007. 17(2): p. 97-114.

48. Chang, Z., et al., Developmental twin study of attention problems: high heritabilities throughout development. JAMA psychiatry, 2013. 70(3): p. 311-318.

49. Hart, S.A., et al., Exploring how symptoms of attention-deficit/hyperactivity disorder are related to reading and mathematics performance: General genes, general environments. Psychological Science, 2010. 21(11): p. 1708-1715.

50. Pingault, J.B., et al., Developmentally dynamic genome: Evidence of genetic influences on increases and decreases in conduct problems from early childhood to adolescence. Sci Rep., 2015. 5:10053.(doi): p. 10.1038/srep10053.

51. Tucker-Drob, E.M. and D.A. Briley, Continuity of genetic and environmental influences on cognition across the life span: a meta-analysis of longitudinal twin and adoption studies. Psychol Bull, 2014. 140(4): p. 949-79.

52. Burt, S.A., Rethinking environmental contributions to child and adolescent psychopathology: a meta-analysis of shared environmental influences. Psychol Bull, 2009. 135(4): p. 608-37. 
53. Merwood, A., et al., Different heritabilities but shared etiological influences for parent, teacher and self-ratings of ADHD symptoms: an adolescent twin study. Psychol Med, 2013. 43(9): p. 1973-84.

54. Derks, E.M., et al., Genetic and environmental influences on the relation between attention problems and attention deficit hyperactivity disorder. Behav Genet, 2008. 38(1): p. 11-23.

55. Groen-Blokhuis, M.M., et al., Attention-deficit/hyperactivity disorder polygenic risk scores predict attention problems in a population-based sample of children. $\mathrm{J}$ Am Acad Child Adolesc Psychiatry, 2014. 53(10): p. 1123-9 e6.

56. Chen, W., et al., DSM-IV combined type ADHD shows familial association with sibling trait scores: a sampling strategy for QTL linkage. Am J Med Genet B Neuropsychiatr Genet., 2008. 147B(8): p. 1450-60. doi: 10.1002/ajmg.b.30672.

57. Martin, J., et al., Genetic risk for attention-deficit/hyperactivity disorder contributes to neurodevelopmental traits in the general population. Biol Psychiatry, 2014. 76(8): p. 664-71. 